\title{
Solvent Structure at a Hydrophobic Protein Surface
}

Helena Kovacs, Alan E. Mark, and Wilfred F. van Gunsteren*

Laboratory of Physical Chemistry, Swiss Federal Institute of Technol ogy Zürich, ETH-Zentrum, Zürich, Switzerland

\begin{abstract}
The impact of an extensive, uniform and hydrophobic protein surface on the behavior of the surrounding solvent is investigated. In particular, focus is placed on the possible enhancement of the structure of water at the interface, one model for the hydrophobic effect. Solvent residence times and radial distribution functions areanalyzed around three types of atomic sites (methyl, polar, and positively charged sites) in 1 ns molecular dynamics simulations of the $\alpha$-helical polypeptide SP-C in water, in methanol and in chloroform. For comparison, water residence times at positively and negatively charged sites are obtained from a simulation of a highly charged $\alpha$-helical polypeptide from the protein titin in water. In the simulations the structure of water is not enhanced at the hydrophobic protein surface, but instead is disrupted and devoid of positional correlation beyond the first solvation sphere. Comparing solvents of different polarity, no clear trend toward the most polar solvent being more ordered is found. In addition, comparison of the water residence times at nonpolar, polar, positively charged, or negatively charged sites on the surface of SP-C or titin does not reveal pronounced or definite differences. It is shown, however, that the local environment may considerably affect solvent residence times. The implications of this work for the interpretation of the hydrophobic effect are discussed. Proteins 27:395-404, 1997. (c) 1997 Wiley-Liss, Inc.
\end{abstract}

Key words: hydration; solvation; protein-solvent interactions; molecular dynamics; computer simulation; GROMOS; SPC water; radial distribution function; solvent residence times

\section{INTRODUCTION}

Calorimetric studies show that the unfolding of a protein in aqueous solution is accompanied by a large rise in the heat capacity, $\Delta \mathrm{Cp}$ (see refs. 1-3, and references therein). The change in heat capacity is usually attributed to a loss of entropy. Empirically this loss of entropy has been shown to be roughly proportional to the increase in nonpolar surface area exposed to solvent. ${ }^{1}$ Therefore, it has been argued that the physical reason for the phenomenon is that water in contact with a hydrophobic atomic surface becomes more ordered, in analogy with the solvation of small mol ecules. ${ }^{1-4}$ A concept of hydrophobic hydration has been developed according to which the exposure of a section of hydrophobic surface of a protein results in the "freezing" of the surrounding water, giving riseto semistable clusters or the formation of so called clathrate structures. ${ }^{5}$ Such structures are envisaged to arise because the absence of polar or hydrogen-bonding interactions to apolar protein atoms enhances water-water interactions. If such ordering of water does in fact occur, it would be predicted that water molecules in close proximity to an exposed hydrophobic surface of a protein would display enhanced (long-range) structure, longer residence times, and slower motions compared to the waters close to a predominantly polar or charged surface. Unfortunately experimental data against which such models can be tested, is scarce.

In crystallographic x-ray studies of proteins, a number of water molecules can usually be resolved at the crystallized protein surface. Therefore, one approach to investigate water structure at protein surfaces is to look for preferred solvation sites. ${ }^{6}$ Surface waters are not, however, completely conserved between different crystal forms.,7 A further complication is that the identification of hydration sites is subjective. There is also a bias towards finding immobilized waters with a high site occupancy. ${ }^{7}$ Nuclear magnetic resonance (NMR) has also been used to characterize protein hydration at individual surface sites. ${ }^{8}$ Because of experimental factors, such as low sensitivity due to short residence times of the surface waters or proximity of hydrogens in rapid exchange with bulk water, ${ }^{9}$ only a minor fraction of surface waters can be unambiguously identified by NMR methods. Specific sites where waters are observed frequently do not corre-

Abbreviations: BPTI, bovine pancreatic trypsin inhibitor; MD, molecular dynamics; NMR, nuclear magnetic resonance; SPC, simple point charge water model; SP-C, surfactant protein C.

Dr. Kovacs is currently at the Department of Biochemistry, University of Oxford, South Parks Road, Oxford OX1 3QU,, U.K.

*Correspondence to: Dr. Wilfred F. van Gunsteren, Laboratory of Physical Chemistry, Swiss Federal Institute of Technology, Zürich, ETH-Zentrum, Switzerland.

Received 16August 1996; accepted 5 September 1996. 
spond to crystal hydration sites. NMR analysis has nevertheless shown that water residence times at the surface of a protein are in the subnanosecond range. $^{9}$

In the light of the experimental difficulties a number of workers have turned to theoretical methods. Molecular dynamics simulations, for example, can provide information on the time scale and at the atomic resolution appropriate for examining protein solvation. Solvent dynamics and/or structure has been analysed in several calculations performed with different force fields. ${ }^{10-22}$ The diffusion of solvent has been shown to decrease in the proximity of the protein ${ }^{11-13,18,19,22}$ in accordance with experimental observations. ${ }^{23}$ This has been interpreted in terms of solvent ordering near the protein, although it may be partly due to the protein surface simply acting as a wall. ${ }^{11}$

Both experimental data and theoretical calculations indicate that the surface of the protein does not influence the solvent uniformly. There has been a number of attempts to correlate solvent molecule behavior with the character of the neighboring protein atoms. In an analysis of the hydration of bovine pancreatic trypsin inhibitor, BPTI, Brunne and coworkers ${ }^{17}$ found the longest residence times for water molecules near polar side chain atoms, followed by those in the vicinity of nonpolar and charged atomic sites. However, a comparison be tween water residence times estimated from NMR and the results from the simulations did not show a clear correlation. ${ }^{17}$ Brooks and Karpl us ${ }^{13}$ found, based on diffusion constants determined for water at nine specific sites in lysozyme, that water mobility was reduced most at apolar sites, followed by charged, then polar sites compared to bulk water. In these two studies different force fields were used for both the protein and the solvent. This fact probably accounts for at least part of the discrepancy.

A major difficulty in simulation studies involving globular proteins is that the local effects on the surrounding solvent are modified by the solvent accessibility of a particular site and interactions with neighboring atoms. Variations in the shape of the radial distribution functions are found even between similar sites, for example, the two methyl groups of a given valine residue. The average residencetimes in BPTI ${ }^{17}$ for nonpolar, polar and charged protein sites all lie within the rms fluctuation of one another. The trend found for the solvent diffusion coefficients around lysozyme ${ }^{13}$ likewise was based on two or three examples of each type of site and the actual values were strongly scattered.

We have previously reported on a series of molecular dynamics simulations of the lung surfactant polypeptide SP-C in chloroform, methanol, and water ${ }^{24}$ in which the effect of the solvent environment on the stability of this integral membrane helix was investigated. The $\alpha$-helix in SP-C, residues ASN9 to
GLY34, exposes an extensive hydrophobic surface to solvent. The 35-residue polypeptide sequence consists of mainly branched, nonpolar residues. The helix contains in particular a nearly uniform lipophilic segment of over $20 \AA$ in length comprising residues LEU 13-VAL28. The nonpolar side chains make approximately $80 \%$ of the total solvent accessible surface, while the exposure of polar side chains and the main chain contribute around $10 \%$ each. SP-C thus represents a unique test case to investigate the solvent structure near a hydrophobic surface.

For the present investigation, solvent residence times $\left(\tau_{\mathrm{rt}}\right)$ were determined at different protein surface sites as these are a measure of the solvent mobility and reflect the strength of the intermolecular interactions. Radial distribution functions were calculated to obtain information on the extent of solvent structure and the preferred molecular orientation at protein surface sites. The high number of valines (13) and leucines (8) in SP-C ensures good statistics of the solvent behavior at the nonpolar sites. Such is not the case, however, with the polar and charged sites, as the SP-C sequence contains only few such residues. In order to enable comparisons of solvent residence times at different types of sites we have included hydration data from a simulation of a predominantly $\alpha$-helical polypeptide from the muscle protein titin (connectin). The 38-residue sequence of this polypeptide contains a total of 10 glutamates and 7 lysines. Hydration around the charged side chain groups of these residues was analyzed.

\section{Simulations \\ COMPUTATIONAL METHODS}

The GROMOS simulation package with the GROMOS87 force field ${ }^{25}$ was used. Data were extracted from two 1.0 ns trajectories of SP-C in chloroform and methanol, respectively, one 1.0 ns trajectory of $\mathrm{SP}-\mathrm{C}$ in water, with an $\mathrm{N}$-terminal 5-residue del etion to reduce the size of the system and hence improve computational efficiency, and one 0.4 ns trajectory of the titin helix in water. The temperature was $300 \mathrm{~K}$. Computational details of the SP-C simulations have been published earlier. ${ }^{24}$ The simulation parameters for the titin peptide were the same as for the truncated SP-C in water. The simulation was performed in a periodic box of truncated octahedron shape with a diameter of $6.47 \mathrm{~nm}$. The sequence of SP-C is LRIPCCPVNLKRLLVVVVVVVLVVVVIVGALLMGL ${ }^{26}$ and that of the titin peptide is KDESYEELLRKTKDE LLHWTKELTEEEKKALAE EGKIT. ${ }^{27}$ Chloroform was described by the model of Dietz and Heinzinger, ${ }^{28,29}$ methanol by the optimized model (OM2) of Stouten 30 with constraints, and water by the simple point charge (SPC) model of Berendsen and colleagues. ${ }^{31}$ The ratio of the size of water, methanol, and chloroform molecules is 
TABLE I. Exclusion Radius $\left(r_{\text {excl }}\right)$ Determined From the Radial Distribution Function and Radius of the Hydration Shell $\left(r_{\text {shell }}\right)$ Used in the Residence Time Calculations*

\begin{tabular}{|c|c|c|c|c|c|c|}
\hline & \multicolumn{2}{|c|}{$\begin{array}{l}\text { Watera } \\
\text { (oxygen) }\end{array}$} & \multicolumn{2}{|c|}{$\begin{array}{c}\text { Methand } \\
\text { (carbon) }\end{array}$} & \multicolumn{2}{|c|}{$\begin{array}{c}\text { Chloroform } \\
\text { (carbon) }\end{array}$} \\
\hline & $r_{\text {exd }}$ & $r_{\text {shell }}$ & $r_{\text {exd }}$ & $r_{\text {shell }}$ & $r_{\text {exd }}$ & $r_{\text {shell }}$ \\
\hline $\begin{array}{l}\text { Side chain methyl } \\
\text { carbon }\end{array}$ & 0.28 & 0.45 & 0.29 & 0.48 & 0.32 & 0.55 \\
\hline Explicit hydrogen & 0.15 & 0.30 & 0.21 & 0.40 & 0.27 & 0.50 \\
\hline Carbonyl oxygen & 0.25 & 0.40 & & & & \\
\hline
\end{tabular}

*Distances are in nanometers.

aValues for water were taken from Brunne et al. ${ }^{17}$

1.0:2.0:4.4 and their dipole moments are 2.3, 2.3, and $1.1 \mathrm{D}$ in the simulation models.

\section{Radial Distribution F unctions}

Pair radial distribution functions between a nominated atom of the solute and a given atom of the solvent were calculated in a half-sphere the radius of which projected al ong a line between the nominated solute atom and the center of mass of its directly bonded neighbors. To interpret the radial distribution functions presented later, it should be noted that the methyl group is defined as a united atom with radius $0.198 \mathrm{~nm}$ in the GROMOS87 force field and that explicit hydrogens have no van der Waals radius. Van der Waals interactions between protein and solvent atoms are computed as the geometric mean of the self interaction parameters $C_{6}$ and $C_{12}$, for example, $C_{6}(i, j)=\left[C_{6}(i, i) C_{6}(j, j]^{1 / 2}\right.$. A value of $C_{12}$ $(\mathrm{OW}, \mathrm{OW})^{1 / 2}=793.3\left[\mathrm{kcal} \mathrm{mol} \mathrm{mo}^{-1} \AA^{12}\right]^{1 / 2}$ was employed $^{32}$ for the interaction between a neutral carbon and SPC water oxygen.

\section{Solvent Residence Times}

The solvent residence times were calculated as described by Brunne and coworkers. ${ }^{17}$ If not stated otherwise, solvent molecules are defined in terms of the oxygen in water, the methyl carbon in methanol, and the carbon atom in chloroform. Complete trajectories were analyzed, discarding only the first $30 \mathrm{ps}$ as equilibration. Initially, radial distribution functions were determined at numerous sites on the protein. From these was extracted a mi nimum exclusion distance, $r_{\text {exc }}$, between the protein atom and the solvent atom. A spherical solvation shell with a radius of $r_{\text {shell }} \cong r_{\text {excl }}+d_{\text {solvent }}+\Delta_{\text {rt }}$ was then defined. The parameter $d_{\text {solvent }}$, corresponding to the maximum dimensions of the solvent molecule, was added in order to allow any orientation of the solvent within the solvation sphere. In chloroform $d_{\text {solvent }}$ was set to the carbon-chlorine bond length of 0.176 $\mathrm{nm}$ and in methanol to the carbon-oxygen bond length of $0.143 \mathrm{~nm}$. Positional fluctuations were included through the term $\Delta_{\mathrm{rt}}=0.05 \mathrm{~nm}$. The $\mathrm{r}_{\mathrm{excl}}$ and $r_{\text {shell }}$ values used for water were taken from
TABLE II. Average Residence Times, $\left\langle\tau_{\mathbf{r t}}\right|$ and Standard Deviation (in ps), for the Different Types of Atomic Sites*

\begin{tabular}{|c|c|c|c|c|}
\hline & $\mathrm{n}$ & $\begin{array}{c}\text { Water } \\
\left\langle\tau_{\mathrm{rt}}\right\rangle\end{array}$ & $\begin{array}{c}\text { Methand } \\
\left\langle\tau_{\mathrm{rt}}\right\rangle\end{array}$ & $\begin{array}{c}\text { Chloroform } \\
\left\langle\tau_{\mathrm{rt}}\right\rangle\end{array}$ \\
\hline \multicolumn{5}{|l|}{ Methyl groups } \\
\hline Nonhelical in & & & & \\
\hline $\begin{array}{l}\text { SP-C } \\
\text { Helical V15-V21 }\end{array}$ & 8 & - & $31 \pm 18$ & $15 \pm 4$ \\
\hline $\begin{array}{l}\text { in SP-C } \\
\text { Helical-LEU in }\end{array}$ & 14 & $8 \pm 2$ & $12 \pm 4$ & $17 \pm 4$ \\
\hline SP-C & 14 & $7 \pm 1$ & $11 \pm 4$ & $19 \pm 4$ \\
\hline $\begin{array}{l}\text { Helical-LEU in } \\
\text { titin }\end{array}$ & 10 & $9 \pm 2$ & & \\
\hline All in SP-C & 48 & $8 \pm 2$ & $14 \pm 10$ & $18 \pm 5$ \\
\hline \multicolumn{5}{|l|}{ All VAL and } \\
\hline LEU in BPTIa & 6 & $19 \pm 13$ & & \\
\hline All in BPTIa & 18 & $24 \pm 13$ & & \\
\hline \multicolumn{5}{|l|}{ Polar sidechains } \\
\hline $\begin{array}{l}\mathrm{SP}-\mathrm{C} \\
\mathrm{ASN}^{-\mathrm{NH}_{2}} \text { in }\end{array}$ & 2 & $5 \pm 0$ & $79 \pm 9$ & $11 \pm 1$ \\
\hline \multicolumn{5}{|l|}{$\begin{array}{c}\text { Charged side } \\
\text { chains }\end{array}$} \\
\hline LYS-H+ in SP-C & 3 & $6 \pm 2$ & $11 \pm 1$ & $8 \pm 1$ \\
\hline LYS-H+ in titin & 21 & $7 \pm 2$ & & \\
\hline LYS-H+ in BPTIa & 12 & $11 \pm 5$ & & \\
\hline GLU-O- in titin & 20 & $14 \pm 5$ & & \\
\hline ВРTIa & 4 & $65 \pm 47$ & & \\
\hline
\end{tabular}

*Number of values used for averaging, $\mathrm{n}$, is given.

aF rom Brunne et al., ${ }^{17} 277 \mathrm{~K}$.

Brunne and coworkers ${ }^{17}$ to enable comparison to the earlier study. Values for $r_{\text {excl }}$ and $r_{\text {shell }}$ are given in Table I. The presence of solvent mol ecules within the solvation shell was recorded every $10 \mathrm{ps}$, which yiel ded a distribution of residence times. An effective residence time, $\tau_{r t}$, was obtained as the decay coefficient of an exponential function that was fitted to the residence time distribution. Average solvation numbers within the solvation shell defined by $r_{\text {shell }}$ were al so determined.

\section{Solvent Residence Times \\ RESULTS}

Table II presents the average residence times at various types of protein methyl sites, at one polar site (ASN), at one positively charged (LYS) and one negatively charged (GLU) side chain group. Data are reported for the SP-C and titin helices. Corresponding water residence times from the BPTI simulation of Brunne and colleagues ${ }^{17}$ are included for comparison. It should be noted that the calculations of Brunne and colleagues ${ }^{17}$ were performed at $277 \mathrm{~K}$. In addition, a more attractive interaction parameter between water oxygen and neutral protein carbon than in the current work was used as well as a shorter long-range cutoff radius ( $1.2 \mathrm{~nm}$ vs $1.4 \mathrm{~nm}$ ). 


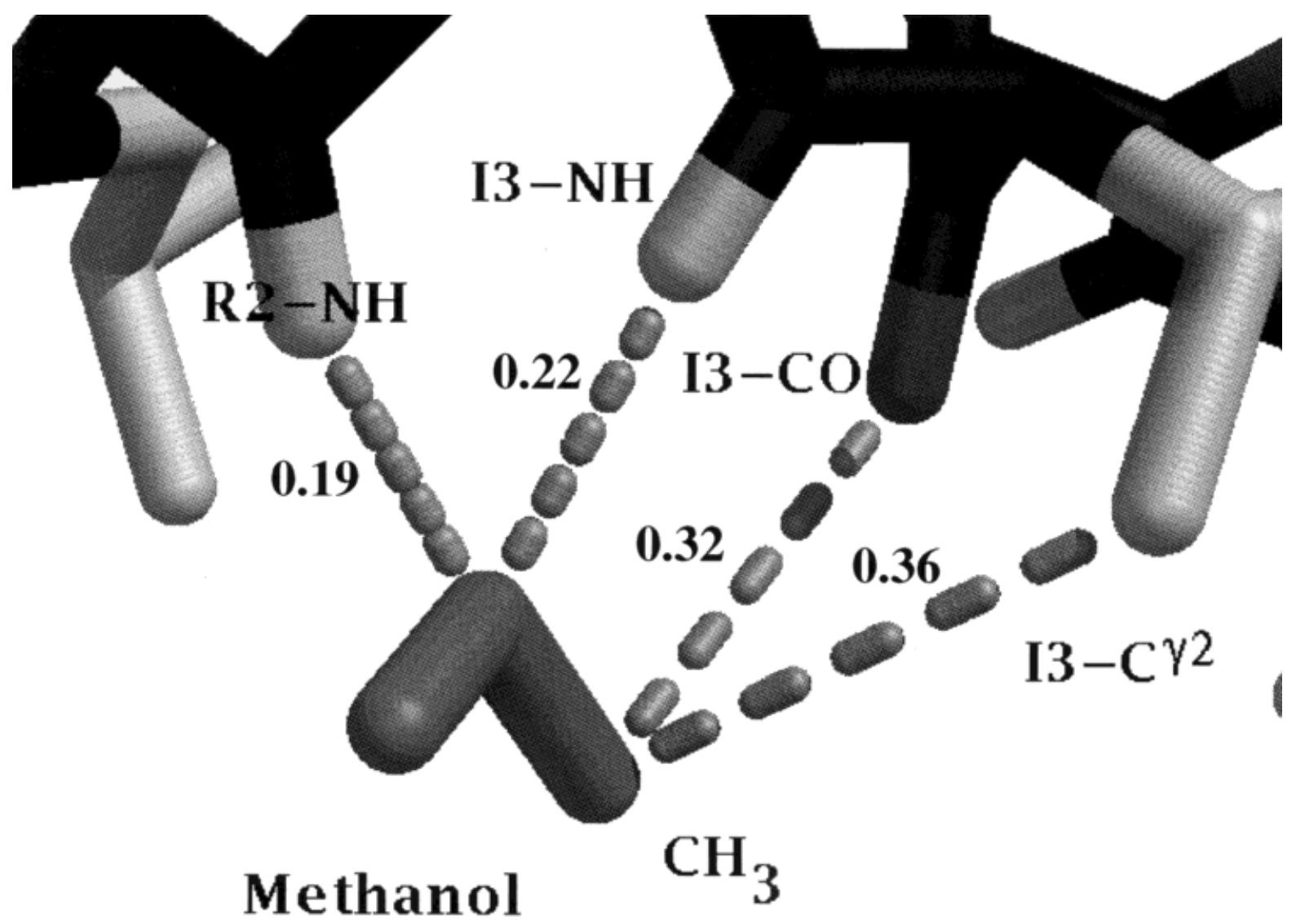

Fig. 1. Snapshot from the simulation of SP-C in methanol at $840 \mathrm{ps}$. The methanol molecule with the longest residence time at the ILE3- $\gamma$-methyl group is within hydrogen bonding distance to the backbone amide groups of ARG2 and ILE3. Distances in nanometers.

Thus, the absolute residence times cannot be compared directly.

\section{Methyl sites}

No significant difference between the average residence times of water molecules located next to the methyls of helical leucines in SP-C (7 ps) or in the titin helix (9 ps) was observed. The mean residence time of water at the valyl-rich surface was $8 \mathrm{ps}$. Due to the $\mathrm{N}$-terminal deletion no value for the residence time of water at nonhelical methyl groups of SP-C can be given. In chloroform average solvent residence times at different methyl sites are again equivalent within statistical uncertainty. In methanol the average solvent residence time is longer near nonhelical methyl groups (31 ps) than near helical ones (11/12 ps). Variation in the methanol values is large. For example, the residence time at the $\gamma$-methyl group of ILE 3 is 60.0 ps while at the $\delta$-methyl group of the sameresidue it is $9.2 \mathrm{ps}$. Theresidence time in such cases is apparently dominated by interactions between the solvent and surrounding protein atoms rather than by theinteraction with the methyl group itself. Figure 1 shows the methanol molecule with the longest life time at the $\gamma$-methyl group of ILE 3 . The solvent molecule is within hydrogen bonding distance from the backbone amide hydrogens of ARG2 and ILE 3.

\section{Polar and charged sites}

The residence times of water from the SP-C and titin simulations at both the positively charged side chain amine hydrogens of lysine (6/7 ps) and at the polar sites of asparagine side chain amide-hydrogens (5 ps) are similar to the average residence time at the methyls (8 ps). This is surprising considering the electrostatic interactions at these sites. The side chain nitrogens of lysine and asparagine both act as a hydrogen bond donors. Lysine carries a formal charge (lysine: CE +0.127e N +0.129e, H +0.248e) compared with asparagine, which is polar (asparagine: $\mathrm{CG}+0.38 \mathrm{e}, \mathrm{O}-0.38 \mathrm{e}, \mathrm{N}-0.83 \mathrm{e} \mathrm{H}+0.415 \mathrm{e}$ ). It might be expected that water would interact more strongly with lysine than with asparagine. The high mobility of lysine can be expected, however, to favor shorter solvent residence times. It should also be noted that the asparagine value is based on only a single residue. The water residence time at the 


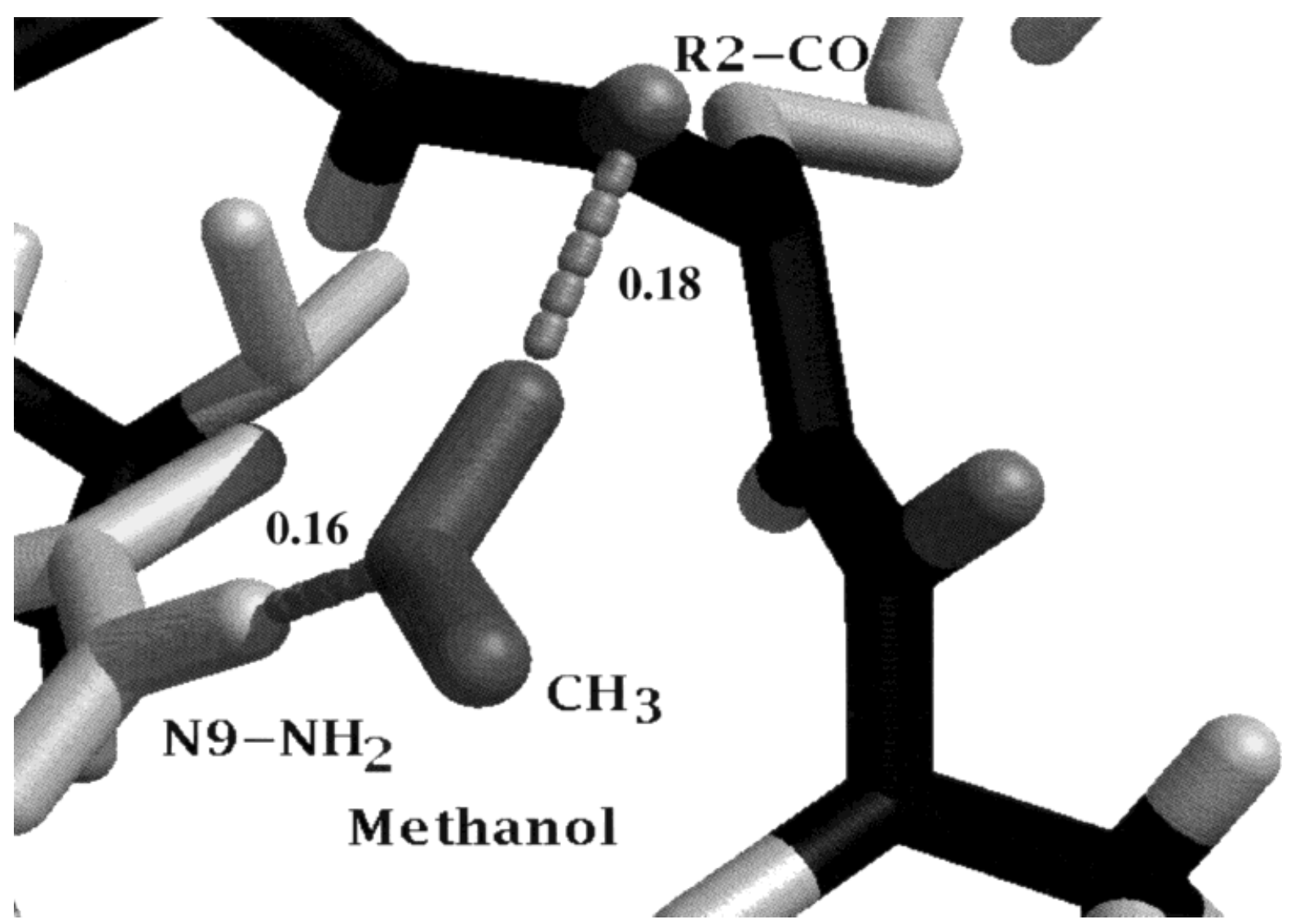

Fig. 2. Snapshot from the simulation of SP-C in methanol at $90 \mathrm{ps}$. The methanol molecule with the longest residence time at the ASN9 side chain amide group is within hydrogen bonding distance to the backbone carbonyl group of ARG2. Distances in nanometers.

negatively charged glutamate-oxygen $(C D+0.27$, $\mathrm{O}-0.635 \mathrm{e})$ was longer at $14 \mathrm{ps}$.

In their simulation of BPTI Brunne and colleagues ${ }^{17}$ found that the mean residence times of water at methyl (24 ps) and polar sites (22 ps) were similar. The mean residence time at the positively charged lysine was comparatively short (11 ps), while the mean residence time at the negatively charged glutamate was very long ( 65 ps) with a large statistical uncertainty. This very likely reflects heterogeneity of the BPTI surface where the residence times are determined by a number of local factors.

From the simulation of SP-C in methanol short residence times were observed at the positively charged hydrogens of lysine (11 ps) but very long residence times at the polar asparagine (79 ps). Although consistent for the two asparagine hydrogens, the long residence time is again associated with interactions to surrounding atoms. This is illustrated in Figure 2, which shows the methanol molecule with the longest residence time at the ASN 9 site forming a hydrogen bond to the mainchain carboxyl group. In chloroform the residence times at the polar (11 ps) and positively charged sites
(8 ps) are significantly shorter than at the methyl groups (18 ps).

\section{Radial Distribution Functions}

Figures 3, 4, and 5 present solute to solvent radial distribution functions of water, methanol, and chloroform, respectively, at different atomic sites normalized to the bulk density of each solvent. Radial distribution functions for each nonequivalent atom of each solvent are displayed in each row. For chloroform, pair radial distribution functions were calculated for each of the three chlorine atoms separately then averaged. The radial distribution functions of the two hydrogen atoms of water were also averaged. The three columns from left to right contain representative nonpolar, polar, and positively charged sites in SP-C, respectively. In the first column the two methyl groups of VAL 17 are given as examples of a nonpolar atomic site in the middle of the polyvaline region. The middle column corresponds to the two side chain amide hydrogens of ASN9, a polar site. The third column, a positively charged site, corresponds to the three side chain amine hydrogens in LYS11. Figure 6a presents an 
VAL17

ASN9

LYS11

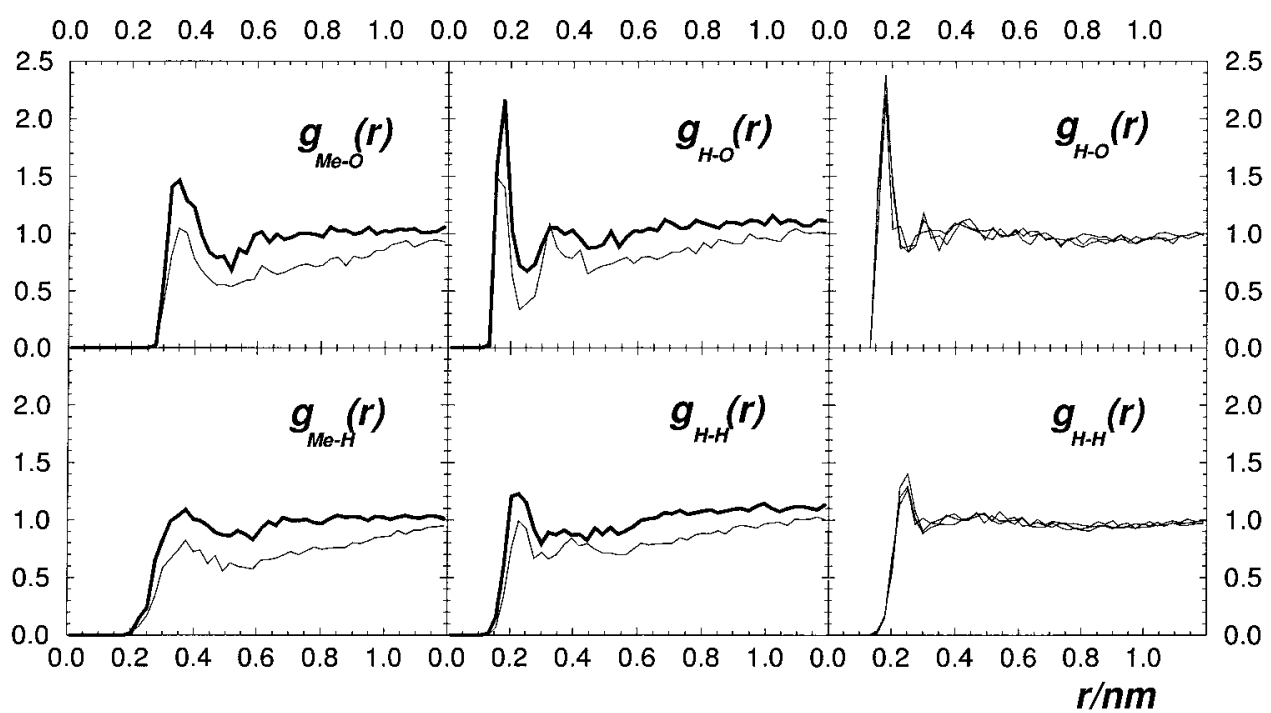

Fig. 3. Solute to solvent radial distribution function of the oxygen (top) and an average of the solute to solvent radial

columns from left to right the solute atoms are the two $\gamma$-methyl groups of VAL17, the two side chain $\delta$ hydrogens of ASN9, and the distribution functions of the two hydrogens of water. In the three

three side chain $\zeta$ hydrogens of LYS11 in SP-C.

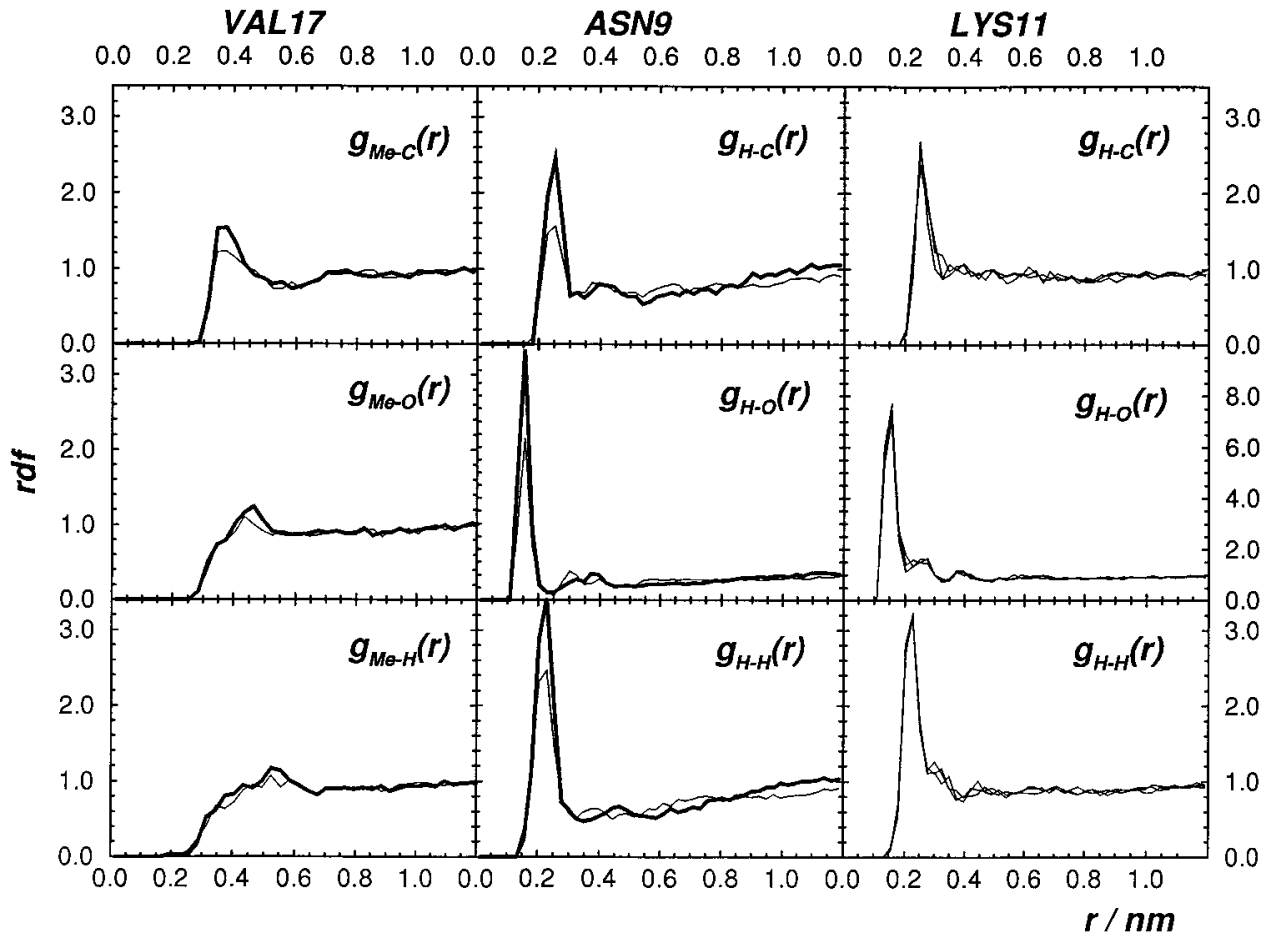

Fig. 4. Solute to solvent radial distribution functions of the carbon (top), oxygen (middle), and hydrogen (bottom) of methanol. In the three columns from left to right the solute atoms are the two $\gamma$-methyl groups of VAL17, the two side chain $\delta$-hydrogens of ASN9, and the three side chain $\zeta$-hydrogens of LYS11 in SP-C.

overlay of the sol ute methyl to solvent radial distribution functions for the water oxygen, the methanol carbon and the chloroform carbon averaged over the 40 methyl groups found in the helical region of SP-C
(ASN9 to GLY34). Figure $6 \mathrm{~b}$ presents an overlay of the solute to water oxygen radial distribution functions, averaged over the 40 methyl groups found in the helical region of SP-C, averaged over the 20 


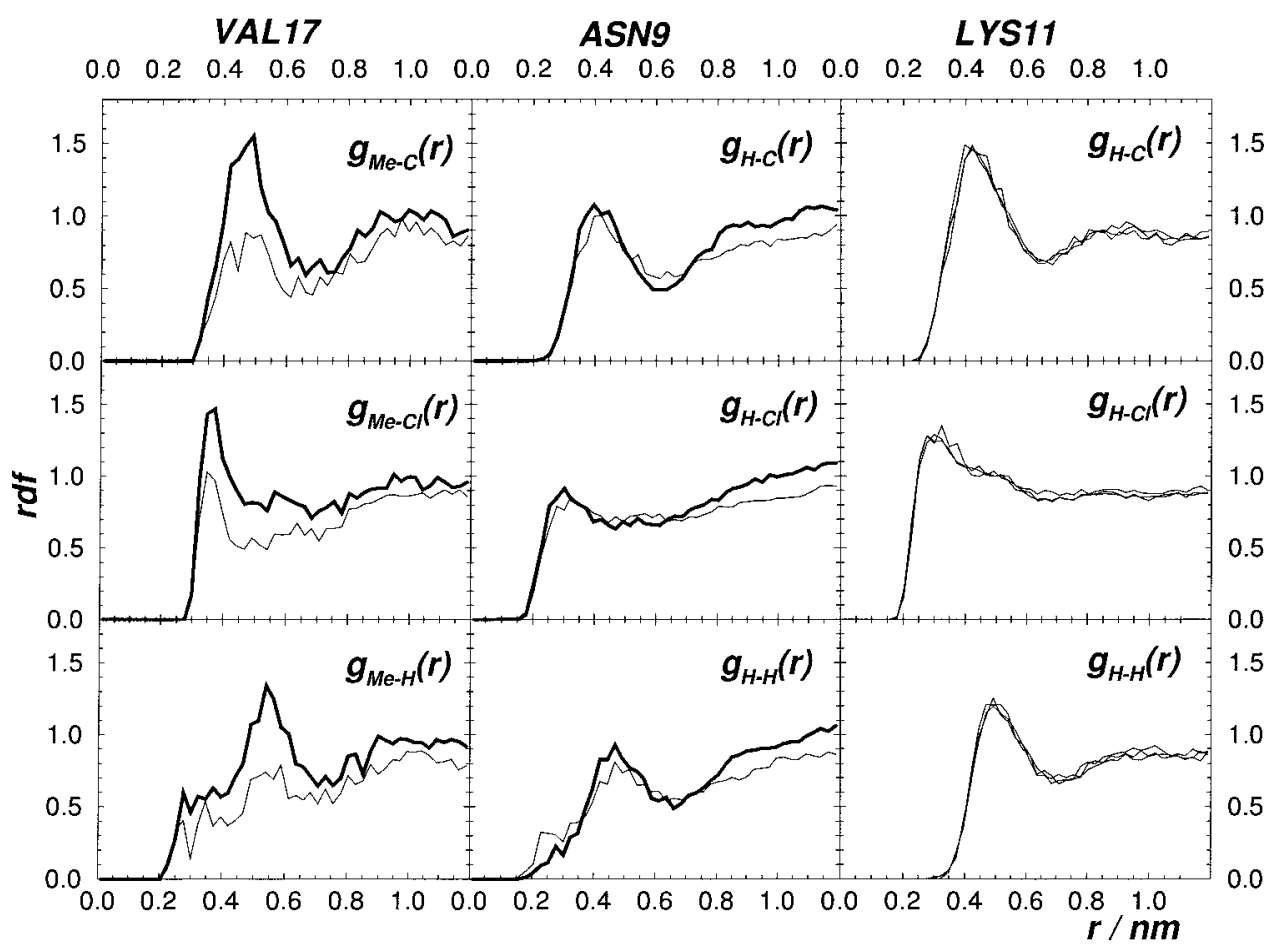

Fig. 5. Solute to solvent radial distribution functions for the carbon (top), chlorine (middle), and hydrogen (bottom) of chloroform. The radial distribution function for the chlorine represents an average over equivalent atoms. In the three columns from left to

glutamate-oxygen sites of titin and averaged over the 21 lysine-hydrogen sites of titin.

\section{Water}

The solute to solvent radial distribution functions of the water oxygen at the methyl sites in Figure 3, first column, display a well-defined peak at $0.35 \mathrm{~nm}$. This distance is equal to the sum of the van der Waals radii of the methyl and oxygen plus the correction for positional fluctuations. The radial distribution function of the water hydrogens display only very broad, flat maxima approximately at the same distance away from the methyl as the water oxygen. This indicates that the hydrogens point into the solution without a well-defined orientation. No evidence for a second hydration shell or long-range ordering of the solvent can be distinguished.

Water approaches both the pol ar and charged sites at a hydrogen bonding distance (Figure 3, middle and right columns). A maximum in the water oxygen radial distribution functions is found at $0.18 \mathrm{~nm}$. The average solvation numbers differ being $1.2 \pm 0.1$ at the polar and $2.0 \pm 0.1$ at the charged site. All the peaks at the ASN9 site are broadened compared to the LYSII site. There is a suggestion of a second peak for oxygen at $0.36 \mathrm{~nm}$ at the ASN site. From Figure $6 \mathrm{~b}$ it is clear that the water is more structured and has greater long-range order at the charged sites than at the nonpolar methyl sites. right the solute atoms are the two $\gamma$-methyl groups of VAL17, the two side chain $\delta$ hydrogens of ASN9, and the three side chain $\zeta$ hydrogens of LYS-11 in SP-C.

It is to be expected that at larger distances the radial distribution functions for the different solute atoms (e.g., the two methyl groups of VAL 17) should converge. To a large extent this is observed. There is, nevertheless, an obvious downward shift in the radial distribution function of one of the two methyl groups of VAL17, and of one of the two hydrogens of ASN9, which persists to $1.0 \mathrm{~nm}$. This shift is a consequence of the manner in which the pair radial distribution functions were defined. Theradial distribution functions were calculated for a half-sphere the radius of which projected along a line between the solute atom in question and the center of mass of its bonded neighbour(s). In the case of the first methyl group in VAL 17 (thick line) the bond from the $\beta$-carbon to the methyl group projects away from the helix directly into the solvent. I n contrast, in the case of the second methyl group (thin line) the bond from the $\beta$-carbon projects at an angle along the helix. This results in the half-sphere containing a lower density of solvent in the second case as compared to the first which causes the observed downward shift in the radial distribution function at short distances. During the simulations in water only a small number of $\chi^{1}$ transitions for the valine residues were observed. This leads to incomplete averaging of the methyl positions. ${ }^{24}$ The same is also true for the two hydrogens of ASN9. One of the two side-chain amide 


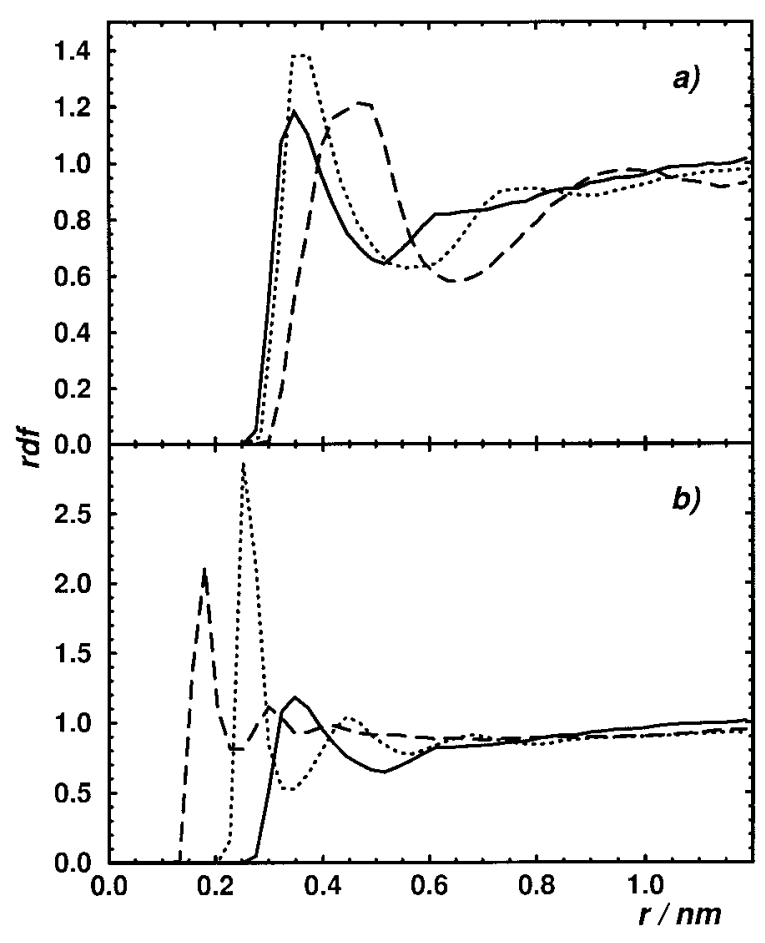

Fig. 6. Solute to solvent radial distribution functions averaged over different atomic sites of the solute. a: The water-oxygen (solid line), the methanol-carbon (dotted line) and the chloroform-carbon (dashed line) to solute-methyl function averaged over the 40 methyl sites in the helical region of SP-C (ASN9 to GLY34). $\mathbf{b}$ : The water-oxygen to different solute atoms function averaged over the 40 methyl sites in the helical region of SP-C (solid line), averaged over the 20 glutamate-oxygen sites of titin (dotted line), and averaged over the 21 lysine-hydrogen sites of titin (dashed line).

hydrogens forms a persistent hydrogen bond with a carbonyl group of the peptide backbone. This restricts the rotation of the ASN9 side chain again resulting in a systematic downward shift of one of the radial distribution functions.

\section{Methanol}

The solute to solvent radial distribution functions in the first column of Figure 4 show that the methanol molecule is preferentially oriented with the methyl group toward the protein methyl sites. A sharp methanol carbon peak is located at contact distance, $0.36 \mathrm{~nm}$, while a broad methanol oxygen peak is at $0.46 \mathrm{~nm}$ and a broad hydrogen peak is found at $0.52 \mathrm{~nm}$. The opposite orientation with the hydroxyl group closer to the methyl group is, nevertheless possible, as indicated by a shoulder on the methanol hydrogen and methanol oxygen radial distribution functions. There is no indication of long-range structure.

The radial distribution functions at the polar and positively charged sites are similar (F igure 4, middle and right columns). The exclusion distance of 0.11 $\mathrm{nm}$ corresponds to the radius of the solvent oxygen. A high, sharp, first maximum is found for the metha- nol oxygen at $0.15 \mathrm{~nm}$. The first maximum is higher at the polar site than at the charged one. This can be explained by the difference in the partial charges, the amide hydrogen in asparagine having a larger value of +0.415 e than the value of +0.248 e on a single amine hydrogen in Iysine. In both cases the methanol methyl group is located further away $(0.26$ $\mathrm{nm}$ ) from the central atom. Thus the exclusion distances and positions of the first maxima are similar for the methanol atoms at the polar and charged sites, showing that the hydrogen-bonding orientation is strongly preferred in both cases. There is a second peak visible in the methanol oxygen radial distribution function. Probably due to steric effects, the second peak at the ASN9 site is at 0.30 $\mathrm{nm}$, while at the LYS11 site it is closer, at $0.26 \mathrm{~nm}$.

\section{Chloroform}

The solute to solvent radial distribution functions for chloroform in the vicinity of VAL 17 given in the first column of Figure 5 show distinct differences between the two methyl groups. At one of the methyl groups (thick line) the orientation of the chloroform molecule is reasonably well defined with the hydrogen preferentially pointing away from the methyl group. There is a broad peak at $0.42-0.52 \mathrm{~nm}$ in the chloroform carbon radial distribution function. In the radial distribution function of the chloroform hydrogen there is a small peak or shoulder at 0.28 $\mathrm{nm}$ corresponding to a contact distance between the protein methyl and the hydrogen and a sharper, more intense, peak at $0.53 \mathrm{~nm}$ corresponding to the more populated orientation with the hydrogen pointing away. At the other methyl group (thin line) the observed preference is much weaker. This probably reflects slight local differences in solvent accessibility. In the final configuration in water, the solvent accessible surface area of the two methyls were 0.39 $\mathrm{nm}^{2}$ and $0.46 \mathrm{~nm}^{2}$. This difference in the exposure is not averaged out in the simulation as this side chain undergoes very few $\chi^{1}$-transitions. Nevertheless, the preferred orientation for chloroform appears to be the one with a chlorine in contact with the protein methyl group.

At the polar site of ASN9 (Fig. 5, middle column) the chlorine radial distribution function is devoid of sharp peaks indicating that the location of the chlorines in the proximity of the polar hydrogens is essentially random. At the positively charged site of LYS11 (Fig. 5, right column) more structure is indicated with the chlorine preferentially oriented toward the amine hydrogens.

\section{DISCUSSION}

An analysis of the simulation of the surfactant protein SP-C in water does not provide evidence for enhanced water structure at the hydrophobic protein surface. The analysis encompasses a large number of methyl sites, which, in the case of the SP-C 
helix, form an extensive and uniform nonpolar surface. Good statistics could be obtained and the scatter of the data is low. Enhanced solvent ordering might be expected to manifest itself by sharper and more intense peaks in the radial solute to solvent distribution functions of water at the protein methyl sites. This was not observed. The presence of second maxima or minima in the radial distribution functions would indicate increased long-range structure relative to that of the pure liquid. No such indications were found. Comparing the results for solvents of different polarity did not show a clear trend toward the most polar solvent being the most ordered. Methanol at the nonpolar sites behaves in a manner similar to water. Chloroform has a preferred orientation at the apolar protein surface and shows some indication of longer range order. Comparison of the water residence times at nonpolar, polar, positively charged or negatively charged sites on the surface of SP-C or titin did not reveal pronounced or definite differences. Chloroform in contrast resides significantly longer around the methyl sites than around the polar or charged sites.

Kuhn and associates ${ }^{6}$ surveyed 56 high-resolution crystal structures and compared surface water sites which were located either on convex regions of the protein surface, the "contact" surface, or on concave regions of the protein surface, grooves, given by the "reentrant" surface. They concluded that intrinsic chemical differences play a role only in the grooves, water having a preference for hydrophilic locations. No clear correlation between the location of water molecules and the type of neighboring protein atom was detected on the flat or convex protein surface in crystals. The surface of the SP-C or titin helix is clearly convex and the current results, showing no dependency of the water residence time on the type of neighboring atom, are consistent with the findings of Kuhn and colleagues. ${ }^{6}$ Wal shaw and Goodfellow ${ }^{33}$ examined the distribution of water sites around apolar side chains in 24 protein crystal structures. They found that a majority (75\%) of the apolar solvent sites were within hydrogen bonding distance of protein polar groups. They proposed that hydrophobic hydration in protein crystals is correlated with the hydration of polar groups and depends on the local environment. In the hydrophobic part of the SP-C helix, solvent access to the backbone is effectively blocked. ${ }^{24}$ Thus, polar interactions between the solvent and the protein main chain that would promote solvent structure at the apolar sites are not possible. In a recent NMR-spectroscopic investigation of human interleukin-1 $\beta$ Ernst and coworkers ${ }^{34}$ observed that all solvent-exposed methyl groups were hydrated. Most of these were not, however, associated with water sites in the three crystal structures. The authors proposed that this was due to the water molecules at hydrophobic sites being positionally disorded. Another possibility was that the water molecules were arranged in substructures with independent motion. Such mobile clusters if present in the simulations might not be detected in the analysis.

It appears that the behavior of the solvent can be strongly influenced by interactions with the surrounding side chains. Compared to the surface of a protein such as BPTI the surface of the SP-C or titin helix is very uniform. The residence times have a standard deviation of $10-20 \%$ at the protein methyls of SP-C, whereas for BPTI ${ }^{17}$ this was approximately $50 \%$ (Table II). In cases where exceptionally long residence times were observed, for example for methanol at $\mathrm{ASN}$ 9- $\mathrm{NH}_{2}$ in SP-C, the long residence time could be associated with the formation of a number a additional polar interactions to the peptide by the solvent molecule. Another indication of local effects is that the radial distribution functions of the two valyl-methyl groups are not equal (Figs. 3-5) reflecting slight differences in local shielding.

In conclusion, from the examination of solvent residence times and radial distribution functions extracted from a series of extended molecular dynamics simulations of a primarily hydrophobic helical peptide in water, in methanol and in chloroform, and of a primarily charged helical peptide in water, we could not detect any clear evidence for the enhancement of the structure of a polar solvent at a hydrophobic protein surface. Obviously, not all structural properties have been investigated and the results will be dependent on the atomic model that has been used. Nevertheless, it is clear that the simulations do not support the very simplistic models of the hydrophobic effect in which the exposure of a nonpolar surface results in the freezing of the motions of the surrounding water molecules.

\section{ACKNOWLEDGMENTS}

Wethank Dr. R. Brunnefor providing the software for the solvent residence times calculations.

\section{REFERENCES}

1. Privalov, P.L., Tiktopulo, E.I., Venyaminov, S. Yu, Griko, Yu. V., Makhatadze, G.I., K hechinashvili, N.N. Heat capacity and conformation of proteins in the denatured state. J Mol Biol 205:737-750, 1989.

2. Creighton, T.E. Stability of folded conformations. Curr. Opin. Struct. Biol. 1:5-16, 1991.

3. Baldwin, R.L. Temperature dependence of the hydrophobic interaction in protein folding. Proc. Natl. Acad. Sci. USA 83:8069-8072, 1986.

4. Karplus, P.A., Faerman, C. Ordered water in macromolecuIar structure. Curr. Opin. Struct. Biol. 4:770-776, 1994.

5. Franks, F. The solvent properties of water. In Franks, F., (ed): "Water: A Comprehensive Treatise," Vol. 2. New York: Plenum, pp 1-54, 1973.

6. Kuhn, L.A., Siani, M.A., Pique, M.E., Fisher, C.L., Getzoff, E.D., Tainer, J .A. The interdependence of protein surface topography and bound water molecules revealed by surface accessibility and fractal density measures. J. Mol. Biol. 228:13-22, 1992.

7. Zhang, X.-J., Matthews, B.W. Conservation of solventbinding sites in 10 crystal forms of T4 lysozyme. Prot. Sci. 3:1031-1039, 1994. 
8. Otting, G., Liepinsh, E., Wüthrich, K. Protein hydration in aqueous solution. Science 254:974-980, 1991.

9. Wüthrich, K., Otting, G., Liepinsh, E. Protein hydration in aqueous solution. Faraday Discuss. 93:35-45, 1992.

10. Berendsen, H.J.C., van Gunsteren, W.F., Zwinderman, H.R.J., Geurtsen, R.G. Simulations of proteins in water. Ann. N.Y. Acad. Sci. 482:269-286, 1986.

11. Wong, C.F., McCammon, J .A. Computer simulation and the design of new biological molecules. Isr. J . Chem. 27:211215, 1986.

12. Ahlström, P., Teleman, O., J önsson, B. Molecular dynamics simulation of interfacial water structure and dynamics in a parvalbumin solution. J. Am. Chem. Soc. 110:4198-4203, 1988.

13. Brooks III, C.L., Karplus, M. Solvent effects on protein motion and protein effects on solvent motion: Dynamics of the active site region of Iysozyme. J. Mol. Biol. 208:159181, 1989.

14. Steinbach, P.J ., Loncharich, R.J ., Brooks, B.R. The effects of environment and hydration on protein dynamics: $A$ simulation study of myoglobin. Chem. Phys. 158:383-394, 1991.

15. Parak, F., Hartmann, H., Schmidt, M., Corongiu, G., Clementi, E. The hydration shell of myoglobin. Eur. Biophys. J . 66:601-614, 1992.

16. Lounnas, V., Pettitt, B.M., Findsen, L., Subramaniam, S A microscopic view of protein solvation. J. Phys. Chem. 96:7157-7159, 1992.

17. Brunne, R.M., Liepinsh, E., Otting, G., Wüthrich, K., van Gunsteren, W.F. Hydration of proteins. A comparison of experimental residence times of water molecules solvating the bovine pancreatic trypsin inhibitor with theoretical model calculations. J. Mol. Biol. 231:1040-1048, 1993.

18. Hartsough, D.S., Merz, K.M. J r. Protein dynamics and solvation in aqueous and nonaqueous environments. J. Am. Chem. Soc. 115:6529-6537, 1993.

19. Komeiji, Y., Uebayasi, M., Someya, J.-i., Yamato, I. A molecular dynamics study of solvent behavior around a protein. Proteins 16:268-277, 1993.

20. Lounnas, V., Pettitt, B.M. A connected-cluster of hydration around myoglobin: Correlation between molecular dynamics simulations and experiment. Proteins 18:133-147, 1994.

21. Lounnas, V., Pettitt, B.M. Distribution function implied dynamics versus residence times and correlations. Proteins 18:148-160, 1994.

22. Norin, M., Haeffner, F., Hult, K., Edholm, O. Molecular dynamics simulations of an enzyme surrounded by vacuum, water, or a hydrophobic solvent. Biophys. J . 67:548-559, 1994.

23. Steinhoff, H.-J., Kramm, B., Hess, G., Owerdreck, C., Redhardt, A. Rotational water diffusion in the Hemogl obin hydration shell: Dielectric and proton nuclear relaxation measurements. Biophys. J . 65:1486-1495, 1993.

24. Kovacs, H., Mark, A.E., J ohansson, J ., van Gunsteren, W.F. The effect of environment on the stability of an integral membranehelix: Molecular dynamics simulations of surfactant protein $\mathrm{C}$ in chloroform, methanol and water. J . Mol Biol. 247:808-822, 1995.

25. van Gunsteren, W.F., Berendsen, H.J .C. Groningen Molecular Simulation (GROMOS) Library Manual. Biomos, Nijenborgh 4, 9747 AG Groningen, The Netherlands, 1987.

26. J ohansson, J ., Curstedt, T., Robertson, B., J örnvall, H. Size and structure of the hydrophobic low molecular weight surfactant-associated polypeptide. Biochemistry 27:35443547, 1988.

27. Musco, G., Tziatzios, C., Schuck, P., Pastore, A. Dissecting titin into its structural motifs: Identification of an alphahelix motif near thetitin N-terminus. Biochemistry 34:553561, 1995.

28. Dietz, W., Heinzinger, K. Structure of liquid chloroform: A comparison between computer simulation and neutron scattering results. Ber. Bunsenges. Phys. Chem. 88:543546, 1984.

29. Dietz, W., Heinzinger, K. A molecular dynamics study of liquid chloroform. Ber. Bunsenges. Phys. Chem. 89:968977, 1985.

30. Stouten, P.F.W. "Small Molecules in the Liquid, Solid and Computational Phases."Dissertation. University of Utrecht, Utrecht, The Netherlands, 1989.

31. Berendsen, H.J .C., Postma, J .P.M., van Gunsteren, W.F., Hermans, J. Interaction models for water in relation to protein hydration. In Pullman, B., (ed): In Intermolecular Forces. Dordrecht: Reidel, pp 331-342, 1981.

32. Mark, A.E., van Helden, S.P., Smith, P.E.,J anssen, L.H.M., van Gunsteren, W.F. Convergence properties of free energy calculations: $\alpha$-Cyclodextrin complexes as a case study. J . Am. Chem. Soc. 116:6293-6302, 1994

33. Walshaw, J., Goodfellow, J .M. Distribution of solvent molecules around apolar side-chains in protein crystals. J . Mol. Biol. 231:392-414, 1993.

34. Ernst, J .A., Clubb, R.T., Zhou, H.-X., Gronenborn, A.M., Clore, G.M. Demonstration of positionally disordered water within a protein hydrophobic cavity by NMR. Science 267:1813-1817, 1995 